$(50 \%)$ of the procedures. None of the consultants or trainees who were involved with these procedures were diagnosed with COVID-19 during this period. One of the nursing staff member, who regularly assisted in the fluoroscopy room, was involved in a non-fluoroscopic diagnostic endoscopic procedure in a COVID-19 patient (not known at the time of the procedure) and subsequently tested positive for COVID-19.

Conclusion Our study confirms that a high quality stenting and dilatation service of the upper gastrointestinal tract together with specialist registrar training can be delivered safely and effectively during the COVID-19 pandemic in appropriately prioritised symptomatic patients.

\section{PTU-28 FACTORS ASSOCIATED WITH SPONTANEOUS PASSAGE OF RADIOLOGICALLY CONFIRMED CBD STONES THROUGH A VIRGIN DUODENAL PAPILLA}

Mohammed Gariballa*, Hussam Ahmed, Rachael Wilkinson-Hall, Amer Al-Joudeh. Sheffield Teaching Hospitals, Sheffield, UK

\subsection{6/gutjnl-2021-BSG.101}

Introduction Spontaneous passage of common bile duct (CBD) stones is a commonly observed clinical phenomenon that eliminates the need for invasive and costly endoscopic retrograde cholangio-pancreatography (ERCP). The aim of this study was to identify factors associated with spontaneous passage of CBD stones before index ERCP.

Methods This retrospective study was conducted in a university teaching hospital. It included patients with a virgin duodenal papilla who underwent ERCP to remove radiologically confirmed CBD stones between April 2018 and October 2019. The primary outcome was the presence/absence of stones on cholangiography where absence indicated spontaneous passage since radiological detection. Data collected retrospectively included patient demographics; scan modality to confirm stones; scan to ERCP time interval, stone number (single vs multiple); largest stone size $(\mathrm{mm})$ and liver function tests (LFT) pre- ERCP.

Student $t$ test was used for comparison of categorical and numerical variables. Chi- Squared test for comparison of categorical variables and Mann Whitney for comparison of the scan to ERCP time intervals. A binominal logistic regression was performed on a subgroup of patients for whom stone size was documented.

Results 427 patients underwent an index ERCP within the study period. Following application of the exclusion criteria 360 patients were included in the final analysis. The absence of a CBD stone on cholangiography was observed in 50 (13.9\%) patients. Mean age was $68.1+/-16.8$ years (Females $52.8 \%)$. CBD stones were confirmed by different imaging modalities (MRCP: 273, CT scan: 66, USS: 21). The presence of a single $\mathrm{CBD}$ stone on imaging was significantly associated with stone passage ;176 (48.9\%) patients had a single CBD stone $(\mathrm{P}<0.05)$. 62\% (31/50) of passed stones were single.

In a subgroup analysis of 142 patients in whom stone size was documented, stone size was found to affect CBD stone passage. Mean stone size of $7.4+/-3.04 \mathrm{~mm}$ was observed in patients with spontaneous passage (15 patients) vs $9.77+$ /$4.76 \mathrm{~mm}$ in those who did not (127 patients), $\mathrm{P}<0.014$. However, on multivariate binominal logistic regression analysis of this subgroup no factors achieved statistical significance including stone size (OR 0.837).
No significant association observed between stone passage and age $(\mathrm{P}=0.108)$, gender $(\mathrm{P}=0.50)$, abnormal LFTs PreERCP $(\mathrm{P}=0.40)$ or the median scan to ERCP time interval [Median of 4 days (IQR 3, 7.25) and 5 days (IQR 3, 24) days respectively $(\mathrm{P}=0.075)$ ].

Conclusions A significant proportion of CBD stones will spontaneously pass. This study suggests solitary stones are more associated with spontaneous passage than multiple stones. A robust prospective study is needed to further investigate if stone size has any significant influence on spontaneous CBD clearance.

\section{PTU-29 UPPER GASTROINTESTINAL BLEEDING AND COVID-19 - A TERTIARY CARE CENTRE EXPERIENCE}

Michael Ding*. On Behalf of OGRES research group (Oesophago Gastrointestinal Researchers into Endoscopy Systems), Queen Elizabeth Hospital Birmingham, Birmingham, UK

\subsection{6/gutjnl-2021-BSG.102}

Introduction Concurrent upper gastrointestinal bleeding (UGIB) in patients with COVID-19 infection has a reported mortality of $21.7 \%$ in a recent study ${ }^{1}$. The threshold for emergency endoscopy was revised during the pandemic in the United Kingdom. We present the largest UK centre dataset to date on upper gastrointestinal bleeding(UGIB) in patients with COVID19 , evaluating incidence and outcomes.

Methods We performed a retrospective cohort analysis of patients treated for COVID-19 (polymerase chain reaction [PCR] positive) from 1/1/20-31/8/20. Patients coded for UGIB (haematemesis/melaena) within this cohort were identified. Binomial logistic regression was used to determine odds ratios of death adjusting for age, sex, UGIB status, Haemoglobin $(\mathrm{Hb})$, heart rate, urea, systolic blood pressure(BP) and body mass index(BMI) (Table 1).

Results 1200 patients were included: 3.8\%(n=46) had UGIB with a median GBS score of 8 (interquartile range [IQR] 613), (Male $=23$, median age 74 [IQR 61-84] years). $26 \%$ $(n=12 / 46)$ of patients with UGIB underwent oesophagogastro-

\begin{tabular}{|c|c|c|c|}
\hline & $\mathrm{OR}$ & $95 \% \mathrm{Cl}$ & $p$ \\
\hline Age & 1.05 & $\begin{array}{l}1.04- \\
1.07\end{array}$ & $<0.001$ \\
\hline Male & 1.20 & $\begin{array}{l}0.89- \\
1.61\end{array}$ & 0.232 \\
\hline $\begin{array}{l}\text { Upper Gl } \\
\text { Bleed }\end{array}$ & 1.03 & $\begin{array}{l}0.48- \\
2.15\end{array}$ & 0.947 \\
\hline $\mathrm{Hb}$ & 0.99 & $\begin{array}{l}0.99- \\
1.00\end{array}$ & 0.139 \\
\hline Heart Rate & 1.02 & $\begin{array}{l}1.01- \\
1.02\end{array}$ & $<0.001$ \\
\hline Urea & 1.05 & $\begin{array}{l}1.03- \\
1.07\end{array}$ & $<0.001$ \\
\hline Systolic BP & 0.99 & $\begin{array}{l}0.99- \\
1.00\end{array}$ & 0.200 \\
\hline BMI & 0.99 & $\begin{array}{l}0.97- \\
1.01\end{array}$ & 0.476 \\
\hline
\end{tabular}

OR: odds ratio; Cl: confidence interval. 
duodenoscopy (OGD). Those who did, had higher red cell transfusion requirements than those who did not (median 1050 [IQR 150-1650] vs 0 [IQR 0-675]ml; p=0.019). 67\% $(8 / 12)$ had oesophageal or peptic ulceration but only $25 \%(2 / 8)$ required endotherapy. There was one re-bleed which required further endotherapy and one required embolisation.

Overall mortality rate in the UGIB cohort is $41.3 \%$. Increased age, heart rate and urea levels were significantly associated with death but not the UGIB status. LOS was prolonged in the UGIB group compared to the non-UGIB group (15 [IQR 8-27] vs 6 [IQR 3-14] days; p<0.001). Subgroup analysis of UGIB patients who underwent OGD compared to those managed conservatively showed no significant difference in mortality (44\%vs 33\%; $\mathrm{p}=0.735$ ) or LOS (12 [IQR 4-28] vs 16 [IQR 8-27] days; $\mathrm{p}=0.349)$.

Conclusions The incidence of concurrent UGIB in patients with COVID-19 infection is low and our data suggests that UGIB does not increase the mortality rate in patients with COVID-19 but does increase LOS. Endoscopic intervention did not appear to affect mortality or LOS. Although numbers are small, this data suggests selection criteria used for endoscopy was justified.

\section{REFERENCE}

1. Mauro A, et al. Upper gastrointestinal bleeding in COVID-19 inpatients: Incidence and management in a multicenter experience from Northern Italy. Clin Res Hepatol Gastroenterol 2020.

\section{PTU-30 INADVERTENT WIRE CANNULATION OF THE PANCREATIC DUCT AT ERCP: A RETROSPECTIVE ANALYSIS OF OUTCOMES}

Ben Arnold, Ben Colleypriest, Rachel Perry*, Benjamin Masterman. Royal United Hosptial, Bath, UK

\subsection{6/gutjnl-2021-BSG.103}

Introduction The procedural risk factors for post-ERCP pancreatitis (PEP) are well documented and include both wire cannulation and contrast injection of the pancreatic duct (PD). Pancreatic guidewire assisted cannulation (PGWAC) is a widely accepted practise and is often employed when common bile duct (CBD) cannulation proves challenging. Given the potential for severe morbidity and mortality from PEP, endoscopists should aim to minimise the risk of this complication and consider prophylactic PD stenting in to addition other measures including rectal non-steroidal administration.

Methods Retrospective data from of all ERCP procedures performed within a 12-month period at a district general hospital were collected. Endoscopy reports were reviewed for indication, diagnosis, CBD cannulation success, the use of needle knife fistulotomy, the number of PD wire passes, deployment of prophylactic pancreatic stent (PPS) and administration of rectal diclofenac. Electronic hospital records were searched for documentation, biochemistry or imaging confirming PEP within 7 days post-procedure.

Results 218 ERCPs were performed in the study period, choledocholithiasis being the most common indication (68.7\%). CBD cannulation success was $89.4 \%$. The overall PEP rate was $2.35 \%$ (95\% CI 1.62-11.67) after excluding 6 cases in which the ampulla was not reached and the risk of PEP was considered negligible. $98 \%$ of cases at which cannulation was attempted received rectal diclofenac and those that didn't had contraindications
In the 212 remaining cases, the PD was inadvertently cannulated in 25 cases $(11.8 \%)$. PEP rates were significantly higher in this group at $12 \%$ compared to $1.1 \%$ if only the CBD was selectively cannulated (RR $11.0495 \%$ CI 1.9382 to $62.88 \mathrm{p}=\mathrm{P}=0.0068) .4$ patients received a prophylactic pancreatic stent after multiple PD wire cannulation and one patient developed mild PEP.

There was a small but statistically insignificant increased risk of PEP if the wire passed in to the PD multiple times rather than a single pass; $13.3 \%$ vs $10 \%$ (RR $1.0385 \mathrm{p}=$ 0.7963). Three patients had injection of contrast into PD with subsequent failed CBD cannulation but none developed PEP.

PWGAC was successful in 18/25 attempts (72\%) and in 7 cases needle knife fistulotomy was attempted with $42.8 \%$ success. There was incidence of PEP in the needled knife group. Conclusions Our data would suggest that any wire cannulation of the PD during ERCP poses a significant risk of PEP. Our outcomes post-PD stenting are, so far, disappointing but metaanalyses $\{1\}$ have demonstrated their benefit in reducing risk of PEP.

Updated meta-analysis of pancreatic stent placement in preventing post-endoscopic retrograde cholangiopancreatography pancreatitis Jin-He Fan, Jun-Bo Qian, Ya-Min Wang, Rui-Hua Shi, and Cheng-Jin Zhao World J Gastroenterol. 2015 Jun 28 ; 21(24): 7577-7583.

\section{PTU-31 OUTCOME FROM A PROSPECTIVE MULTICENTRE TRIAL ON REAL TIME OPTICAL DIAGNOSIS- BLAST STUDY}

${ }^{1}$ Ejaz Hossain*, ${ }^{2}$ Erik Schoon, ${ }^{3}$ Bu Hayee, ${ }^{4}$ Milan Stefanovic, ${ }^{5}$ Saskia Papa, ${ }^{1}$ Mohammed Abdelrahim, ${ }^{1}$ Pradeep Bhandari. 'Portsmouth Hospital Nhs Trust, Portsmouth, UK; ${ }^{2}$ Catharina Hospital, Eindhoven, Netherlands; ${ }^{3}$ Kings College Hospital, London, UK; ${ }^{4} D C$ Bled, Llubjana, Slovenia; ${ }^{5}$ Fujifilm Europe, Germany

\subsection{6/gutjnl-2021-BSG.104}

Introduction Blue Light Imaging (BLI) is a new enhanced imaging technology that enhances mucosal surface and vessel patterns. A specific BLI classification was recently developed to enable better characterisation of colorectal polyps (BLI Adenoma Serrated International Classification - BASIC). We aim to use BLI for real time optical diagnosis during colonoscopy to see if it could meet the PIVI standards.

Methods It is a multicentre, prospective study. All endoscopists were trained in BASIC classification. Patients undergoing colonoscopy with 700 series colonoscopes (Fujifilm Co, Tokyo, Japan) on Eluxeo platform from Fujifilm were recruited in the study. Endoscopists were asked to make real time optical diagnosis of colorectal polyps using WLI and BLI. The accuracy, sensitivity, specificity, and negative predictive value of highconfidence optical diagnosis (adenoma vs. non-adenoma) by either BLI or HDWL for polyps up to $10 \mathrm{~mm}$ was calculated. Results A total of 370 polyps $\leq 10 \mathrm{~mm}$ were detected in 150 patients. The polyp sizes ranged from 1-10mm. $280(75.6 \%)$ polyps were diminutive $(\leq 5 \mathrm{~mm})$ and $255(68.9 \%)$ were adenomas. $131(35.4 \%)$ polyps $\leq 5 \mathrm{~mm}$ were detected in the rectosigmoid. The overall accuracy of BLI compared to WL was $91.9 \%$ vs $88.9 \% \quad(p=0.2)$. Overall $82.4 \%$ polyps were diagnosed with high confidence with BLI compared to $77.4 \%$ with WL. The NPV for diminutive rectosigmoid polyps using BLI was $90.9 \%$ and overall post polypectomy surveillance interval was correctly predicted in $92.5 \%$ patients according to ESGE guidelines. Further analysis of the polyps using BLI is demonstrated in table 1 . 